\title{
Aspectos quirales del grafeno
}

\author{
Graphene chiral elements
}

\author{
H. Torres-Silva ${ }^{1} \quad$ J.L. López-Bonilla ${ }^{2}$ \\ Recibido 22 de octubre de 2010, aceptado 31 de marzo de 2011 \\ Received: October 10, $2010 \quad$ Accepted: March 31, 2011
}

\begin{abstract}
RESUMEN
El grafeno, una alotropía del carbono, es un logro de los avances de la nanotecnología. Siendo una nanoestructura, este componente estructural del grafito posee propiedades que lo han convertido en un potencial sustituto del silicio en el diseño y fabricación de circuitos integrados. Este artículo resume sus orígenes, posibilidades teóricas, así como sus propiedades prácticas. Se presentan aspectos teóricos de quiralidad en el grafeno, específicamente una teoría quiral para el grafeno, vinculando las relaciones constitutivas de Born Fedorov de un campo electromagnético, con las funciones de onda de la ecuación quiral de Dirac para partículas sin masa. Como aplicación se presentan algunos resultados sobre resistencia diferencial negativa, importante en el diseño de osciladores de muy alta frecuencia.
\end{abstract}

Palabras clave: Grafeno, quiralidad, nanoestructuras de carbono, nanociencia, nanotecnología.

\begin{abstract}
The graphene, a carbon allotropy, is a success of the advances of the nanotechnology. Being a nanostructure, this structural component of the graphite possesses properties that have transformed it into a potential substitute of the silicon in the design and production of integrated circuits. This article summarizes its origins, theoretical possibilities, as well as its practical properties. Theoretical aspects of chirality in graphene are presented, specifically a chiral theory for graphene, linking Born Fedorov constitutive relations for an electromagnetic field with the wave functions of the chiral Dirac equation for massless particles. As an application, some results on negative differential resistance are presented, important in the design of very high frequency oscillators.
\end{abstract}

Keywords: Graphene, chirality, carbon nanostructures, nanoscience, nanotechnology.

\section{INTRODUCCIÓN}

El grafeno es una estructura laminar plana, bidimensional del espesor del orden de un átomo [1-6]. La red está compuesta por átomos de carbono densamente empaquetados en dos subredes cristalinas tipo panel de abejas mediante enlaces covalentes que se formarían a partir de la superposición de los enlaces híbridos $\mathrm{sp}^{2}$ de los átomos de carbonos.

El aspecto relevante de esta configuración es que los valores experimentales del grafeno, tales como la conductividad y la absorción óptica, vienen definidos por unidades físicas fundamentales independientemente del origen de la muestra. Difícilmente se puede encontrar un material que haya creado tantas expectativas en la comunidad científica y en tan poco tiempo como el grafeno. Su espectacular auge rivaliza ya con el que en su día tuvieron los nanotubos de carbono, las multicapas magnéticas, las heteroestructuras semiconductoras e incluso con el de los propios superconductores de alta temperatura crítica. El hecho de que su valor experimental venga definido por unidades físicas

\footnotetext{
Instituto de Alta Investigación. Universidad de Tarapacá. Arica, Chile. E-mail: htorres@uta.cl

2 SEPI, Escuela Superior de Ingeniería Mecánica y Eléctrica. Instituto Politécnico Nacional. Zacatenco, México DF.

E-mail: joseluis.lopezbonilla@gmail.com
} 
fundamentales, independientemente del origen de la muestra de grafeno, es algo extraordinario y teóricamente no resuelto.

El grafeno (Figura 1), como elemento estructural componente del grafito es el material más conductor y resistente que existe en la actualidad y estas características lo hacen un futuro sustituto del silicio en la fabricación de chips en la electrónica integrada.

Las propiedades más destacadas del grafeno son:

- Alta conductividad térmica y gran conductividad eléctrica.

- Alta elasticidad, gran dureza y una resistencia mecánica mayor que el acero.

- Químicamente, el grafeno reacciona con otras sustancias para formar compuestos con diversas propiedades. Transformando al grafeno como un material de gran potencial electrotecnológico.

- Presenta alta resistencia a la ionización.

Teóricamente, en el grafeno los electrones se comportan como cuasipartículas, o sea, como partículas cargadas eléctricamente pero con masa igual a cero. Estos cuasifermiones, conocidos como fermiones sin masa, o de Weyl-Dirac, son vitales en la Teoría Relativista. Estos fermiones de Weyl Dirac se mueven a una velocidad constante de aproximadamente $10^{6} \mathrm{~m} / \mathrm{s}$.

Otra propiedad teórica del grafeno es el efecto Hall cuántico, por el cual la conductividad perpendicular a la corriente electrónica toma valores discretos, o cuantizados, significando con esto que la conductividad del grafeno no puede desaparecer. En la actualidad la fabricación de dispositivos flexibles se basa en la utilización de óxido de estaño e indio, que son materiales frágiles y costosos. La natural sustitución por el grafeno impreso es una nueva tecnología más eficiente y barata. Actualmente se diseñan y fabrican transistores de grafeno sin degradación, capaces de funcionar a una frecuencia de corte entre 100 y $300 \mathrm{GHz}$, sólo limitada por la capacitancia parásita del substrato de silicio. Esta frecuencia es comparable a la de los mejores transistores de alta frecuencia.

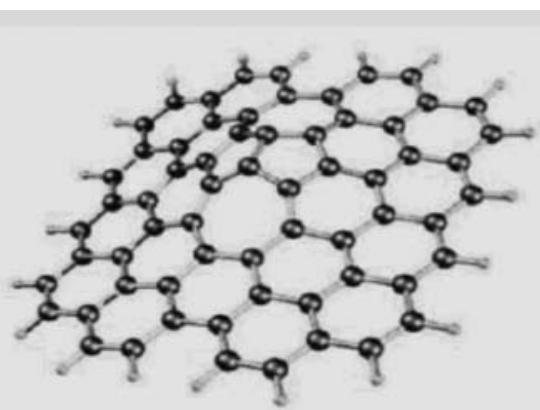

a)

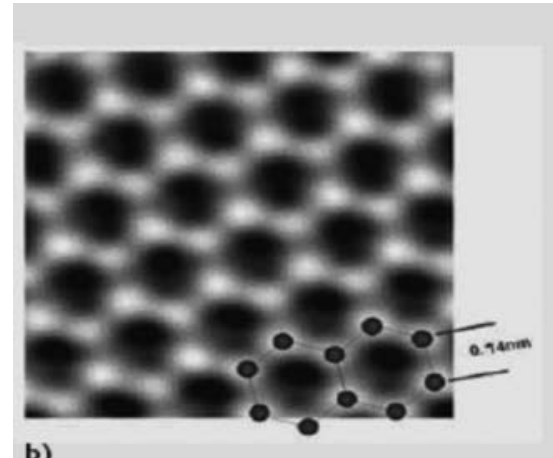

Figura 1. a) Simulación de capas de grafeno, b) grafeno real, imagen de microscopía electrónica de transmisión. Fuente: [2].

\section{DESCRIPCIÓN CUALITATIVA DEL GRAFENO}

\section{Propiedades físicas del grafeno}

La síntesis química del grafeno ha dado hasta el momento sólo pequeñas plaquetas (unos 10 anillos de benceno) que contienen hasta 222 átomos de carbono. Las técnicas convencionales de crecimiento de los cristales también son de poca ayuda, ya que las fluctuaciones térmicas en las temperaturas de crecimiento tienden a girar en tercera dimensión. Los cristales nacientes en 2 Dimensiones tratan de minimizar su energía superficial e inevitablemente se transforman en una variedad de estructuras estables en $3 \mathrm{D}$ que se producen en el hollín.

Pero hay una manera de solucionar el problema. Las interacciones con las estructuras 3D estabilizan los cristales 2D durante el crecimiento. Así que uno puede hacer cristales 2D intercalado entre o sobre la parte superior de los planos atómicos de un cristal grueso. En este contexto, el grafeno ya 
existe dentro del grafito, que puede ser visto como una pila de capas de grafeno pegados por el efecto Van der Waals.

Las hojas de grafeno, como fullerenos y nanotubos de carbono, siempre han existido. Existen incluso en el trazo del lápiz, ya que este trazo está compuesto de gruesos copos de grafito. Normalmente, existen cristales de grafeno de pocas micras de tamaño en un campo de escombros de grafito, cubriendo exactamente una superficie total de $1 \mathrm{~cm}^{2}$.

Para determinar la presencia de grafeno, el laboratorio de Manchester decidió no usar papel, o cualquier otra superficie de escritura, sino una oblea de silicio oxidado, el mismo material utilizado por la industria de los semiconductores. La superficie de óxido refleja un arco iris de colores, y el patrón de interferencia producida por las capas de grafeno en el óxido proporciona un contraste débil, pero visible, al igual que los márgenes en un charco de aceite. A través de este proceso, se obtienen cristales de grafeno en un promedio de dos horas.

En realidad, nadie usa lápices para hacer grafeno. Para hacer cristales de grafeno adecuados para los experimentos se utiliza grafito al granel, el cual se empuja suavemente a lo largo de una oblea de silicio, una técnica que varios laboratorios han refinado al nivel de arte. Los cristales de grafeno producidos llegan a tener hasta $100 \mu \mathrm{m}$ de diámetro.

\section{Relatividad lenta $c \rightarrow v_{F}$}

El grafeno es un semiconductor sin espacios en el que la valencia y la banda de conducción de energía son funciones lineales de impulso. Esta propiedad implica que la velocidad de los electrones en el grafeno es una constante $v_{F}$, independiente del momento, al igual que la velocidad de los fotones es la constante $c$ (velocidad de la luz). De hecho, la mecánica cuántica de los electrones del grafeno es idéntica a la mecánica cuántica relativista de las partículas con una pequeña masa, y son estados libres de partículas quirales.

La ecuación de Dirac nos dice, por ejemplo, que al transformarse en antipartículas virtuales, las partículas relativistas pueden penetrar una barrera potencial de cualquier altura y anchura sin crear un componente paralelo o reflejado. Este efecto, llamado la paradoja de Klein, es uno de los fenómenos más contrario a la intuición de la Electrodinámica cuántica QED.

La velocidad $v_{F}$ de los electrones del grafeno es de aproximadamente $10^{6} \mathrm{~m} / \mathrm{s}$ que es la velocidad de fermi, 300 menor que $c$. Debido a que los electrones son lentos en comparación con los fotones que intercambian durante la interacción, la física de las interacciones electrón-electrón en el grafeno es diferente a la de las interacciones mediadas por fotones entre fermiones en QED.

En el grafeno las interacciones entre los electrones se espera que sean muy fuertes debido a que su selección mutua es más débil que en los metales y porque las dimensiones de acoplamiento de grafeno están definidas por la constante $\alpha=\frac{e^{2}}{\hbar v_{F}} \approx 1$ es mucho más grande que el acoplamiento constante adimensional de QED, definido por la constante de estructura $\alpha=\frac{e^{2}}{\hbar c}=1 / 137$.

La gran diferencia entre c y $v_{F}$ también implica que el modelo que describe la interacción de electrones en una hoja de grafeno, a diferencia de la versión 2D de QED no es una variante de Lorentz. De hecho, las interacciones entre los electrones en el grafeno conducen a un aumento dramático de la velocidad de la partícula.

Para apreciar lo inusual del sistema de fermiones de Dirac, considere su conductividad en el punto de neutralidad, donde la banda de valencia y conducción se encuentran. Puesto que la densidad de los electrones o los agujeros se desvanece cuando la energía de Fermi se aproxima a cero, la expectativa natural es que la conductividad también debe desaparecer en este límite. En este caso particular, el experimento burla esa expectativa.

El grafeno nunca ha mostrado una resistencia mayor a algunos kilo ohms, y los intentos para hacerlo menos conductivo han fracasado hasta ahora. La teoría actual sostiene que cuando la energía de Fermi se acerca a cero, incluso el último electrón o un agujero proporcionan una conductividad mínima de aproximadamente $\frac{e^{2}}{2 \pi \hbar}$, independientemente 
que una hoja de grafeno mida una micra cuadrada o una milla cuadrada.

Parte de lo que explica la conductividad distinta de cero es la dificultad de localizar los fermiones de Dirac sin masa, que pueden deslizarse a través de un potencial paisaje agreste y atrapando a un electrón no relativista. De hecho, los fermiones de Dirac sin masa no se juntan en un espacio potencial 2D hueco, a diferencia de los fermiones Schrödinger que lo hacen, sin importar qué tan débil es el potencial.

Debido a que los electrones de grafeno son tan difíciles de localizar, es tentador recurrir a un viejo argumento de Nevill Mott: Como la luz, un electrón en un metal no distingue ningún tipo de rugosidad en una escala más corta que su longitud de onda $\lambda$ $\mathrm{y}$ por lo tanto nunca puede tener un camino libre menor $l$ que $\lambda$. Estableciendo $l \approx \lambda$ entonces, la teoría de Boltzmann de transporte estándar implica que $\left(e^{2} / h\right) l / \lambda \approx e^{2} / h$.

\section{Variaciones Cuánticas tipo Hall}

Este efecto nos permite hacer inferencias sobre el espectro electrónico de la base de datos de transporte en función de portador de la densidad $\mathrm{n}$ de carga. Nótese que en cero el campo magnético de los estados no existe en esta energía. Por otra parte, sabemos por nuestra experiencia con el efecto Quantum Hall que los valores de la meseta de conductividad transversal $\sigma$ miden la densidad en la que los niveles Landau, están llenos.

Las mesetas en $\sigma= \pm 2 e^{2} / h$ indican que el nivel $\mathrm{E}=0$ toma sólo la mitad del número habitual de los estados de la banda de conducción y señala a la otra mitad de la banda de valencia. Esta característica inusual de Landau nivel cero es responsable de la secuencia anómala de mesetas $\sigma$ en el grafeno. La secuencia de los niveles de Landau se desplaza por un medio, en comparación con el efecto Hall cuántico estándar, de modo que $\sigma= \pm 4 e^{2} / h(N+1 / 2)$, donde $\mathrm{N}$ es el índice de nivel de Landau y el factor de 4 representa el giro del grafeno.

El efecto Hall cuántico bicapa en grafeno es aún más interesante. El campo magnético atrae dos niveles de Landau a $\mathrm{E}=0$, uno de la banda de conducción y otro de la banda de valencia. Las mesetas aparecen ahora en valores enteros de $4 e^{2} / h$, pero es necesario realizar saltos por cada dos unidades al cruzar el nivel de Landau vinculado al punto neutral. Esta cuantificación inusual de bicapa en el grafeno conduce a nuevas alteraciones elementales llamadas fermiones masivos de Dirac.

Los fermiones tienen dispersión cuadrática, como partículas masivas, pero son quirales y se describe por una diagonal externa, de tipo Dirac-Hamilton.

Uno de los triunfos de la ecuación de onda relativista de Dirac fue que proporcionó una explicación del factor $\mathrm{g}$ de electrones, que es igual a 2 si pequeñas correcciones QED se ignoran, debido a que el momento angular de un electrón en el vacío es igual, mientras que los estados orbitales con energías diferentes difieren en el momento angular de los valores enteros. Un factor $\mathrm{g}=2$ implica que la división de energía de giro es igual a la de división de energía orbital. Cuando el grado de libertad de rotación es contado, cada estado orbital se divide en pares, donde uno de los elementos de cada par cambió en la energía y el otro es desplazado hacia abajo.

Sin embargo, debido a que el giro y desdoblamientos orbitales son idénticos, todos los estados permitidos por casualidad continúan siendo doblemente degenerados, a excepción de los niveles más bajos de electrones y de positrones en el nivel más alto, que se dividen por $2 m c^{2}$. Esta cantidad es el doble de la energía en reposo.

Por último, otro aspecto interesante es la observación de que en campos magnéticos lo suficientemente fuertes, se abren brechas adicionales en el espectro de grafeno a niveles de Landau parciales. Estas diferencias son consecuencia de las simetrías rotas. El ejemplo más interesante es la meseta adicional que aparece en una capa eléctrica neutra de grafeno debido a la polarización espontánea que ocurre en el semi lleno nivel Landau de $\mathrm{N}=0$.

El grafeno es el primer ejemplo de materia 2D cristalina. Debido a que el grafeno puede prepararse como una hoja suspendida, la física exuberante de las membranas blandas ahora puede ser explorada en nuevas formas.

Las frecuencias de resonancia mecánica de una membrana suspendida son, como los de una cabeza de tambor, proporcionales a la raíz cuadrada de la 
tensión aplicada. Cuando se suspende de manera muy ligera, las membranas biológicas y otras láminas delgadas se alteran y tienden a arrugarse. Este efecto ocurre a consecuencia del allanamiento del grafeno, debido a que las variaciones lentas en la orientación de la membrana corresponden a rotaciones que consumen muy poca energía.

En resumen, el principal motivo del interés despertado por este material es la naturaleza excepcional de sus portadores. En Física de la Materia Condensada, la ecuación de Schrödinger describe las propiedades electrónicas de cualquier material. El grafeno no es una excepción pero las interacciones con los núcleos de los átomos de carbono hacen que los electrones en el grafeno imiten en su comportamiento a partículas relativistas sin masa gobernadas por la ecuación de Dirac. Si a la falta de defectos estructurales y consiguiente estabilidad mecánica le añadimos las mencionadas propiedades electrónicas "relativistas", el resultado es un material en el que sus portadores muestran una movilidad extraordinaria si se compara con la de semiconductores como el silicio. Se cree que la movilidad está fundamentalmente limitada por impurezas derivadas del contacto con el sustrato, aunque, a día de hoy, la naturaleza de éstas no haya sido identificada con certeza. Otro aspecto aún más intrigante, es el hecho de que la conductividad del grafeno sin dopar es finita y aparentemente está definida a través de constantes universales teniendo un valor en torno a $4 e^{2} / h$ para la mayor parte de las muestras. Esto es algo difícilmente anticipable dado que el grafeno es un semiconductor de gap cero y su densidad de estados es cero al nivel de Fermi. ¿Por qué el valor de la conductividad es universal y cuál es su origen microscópico? ¿Por qué el valor de la velocidad es la velocidad de Fermi y no la velocidad de la luz que es universal que se encuentra en la genuina ecuación de Dirac? Estas son preguntas fundamentales que permanecen sin respuesta. En este trabajo damos respuesta a la segunda pregunta a través de la quiralidad del campo electromagnético en el interior del grafeno.

En este trabajo se presenta la caracterización de medios quirales, a través del formalismo de Born Fedorov y de Pasteur, estableciéndose una equivalencia que permite estudiar y analizar el comportamiento de los parámetros de quiralidad y de dispersión espacial. En la sección siguiente se deriva la ecuación bi dimensional de Dirac de acuerdo al modelo de Born Fedorov.

\section{MODELO MATEMÁTICO DE MEDIO QUIRAL PARA EL GRAFENO}

Los medios quirales pueden ser caracterizados mediante diferentes formalismos matemáticos, de acuerdo a las aplicaciones deseadas. Para nuestros propósitos se utilizan las relaciones constitutivas de Born-Fedorov y Pasteur [7], ya que ellas permiten relacionar la dispersión espacial con la quiralidad. Las ecuaciones de Born-Fedorov están dadas por:

$$
\begin{aligned}
\vec{D} & =\varepsilon_{E D F}(\vec{E}+T \nabla \times \vec{E}) \\
\vec{B} & =\mu_{D B F}(\vec{H}+T \nabla \times \vec{H})
\end{aligned}
$$

Como se aprecia en las ecuaciones (1) y (2), a la relación constitutiva básica se le suma un término rotacional, el que representa la dispersión espacial, cuyos coeficientes son $\varepsilon_{D B F} T$ y $\mu_{D B F} T$, donde $\vec{D}$ es la densidad del flujo eléctrico $\left(\mathrm{C} / \mathrm{m}^{2}\right), \vec{E}$ es la intensidad de campo eléctrico $(\mathrm{V} / \mathrm{m}), \varepsilon_{D B F}$ es la permitividad del medio $(\mathrm{F} / \mathrm{m}), \vec{B}$ es la densidad del flujo magnético $\left(\mathrm{Wb} / \mathrm{m}^{2}\right), \vec{H}$ es la intensidad del campo magnético, $(\mathrm{A} / \mathrm{m}) \mu_{D B F}$ es la permeabilidad magnética $(\mathrm{H} / \mathrm{m})$ y $T$ es el parámetro quiral $(m)$. Debido a que el parámetro quiral tiene unidades de longitud, se le asocia al fenómeno de dispersión espacial. La segunda relación constitutiva, usada en este trabajo, es la de Pasteur, en razón de la relación lineal entre las variables y por ser covariantes. Las ecuaciones de Pasteur son:

$$
\begin{aligned}
& \vec{D}=\varepsilon \vec{E}-i \kappa \vec{H} \\
& \vec{B}=\mu \vec{H}+i \kappa \vec{E}
\end{aligned}
$$

El parámetro $\kappa$ representa la quiralidad del medio y tiene como unidad el recíproco de velocidad $(\mathrm{s} / \mathrm{m})$, su valor puede ser positivo o negativo, lo que dependerá de la estructura quiral; se asume $\kappa>0$. Debido a que tanto Born-Fedorov como Pasteur consideran medios recíprocos, se hace la equivalencia entre ambas relaciones constitutivas, lo que permite establecer, por una parte, una relación entre los parámetros del medio $(\mu$ y $\varepsilon$ ) y la quiralidad $(\kappa)$, y por otra, relacionar la dispersión espacial $(T)$ con la quiralidad $(\kappa)$. 
La relación entre los campos se asume de la forma $\vec{E}=\alpha \vec{B}$, y considerando que $\alpha=i / \sqrt{\mu \varepsilon / 4}$, se obtiene la ecuación $\vec{E}=i \eta \vec{H}=i \sqrt{\frac{\mu / 2}{\varepsilon / 2}} \vec{H}$. Esto permite redefinir la permitividad y la permeabilidad como $\mu / 2$ y $\varepsilon / 2$ respectivamente ([7]):

$$
\begin{gathered}
\varepsilon_{I I}=\varepsilon_{D B F} / 2=\varepsilon\left(1-\frac{\kappa^{2}}{\mu \varepsilon}\right) \\
\mu_{I I}=\mu_{D B F} / 2=\mu\left(1-\frac{\kappa^{2}}{\mu \varepsilon}\right) \\
T=\frac{\kappa}{\omega\left(\mu \varepsilon-\kappa^{2}\right)} T=\frac{\kappa}{\omega\left(\mu \varepsilon-\kappa^{2}\right)}
\end{gathered}
$$

Las ecuaciones (5) y (6) muestran la permitividad y permeabilidad de Born-Fedorov normalizada a los parámetros de Pasteur. Mientras que con la ecuación (7) se obtiene una relación entre la dispersión espacial y la quiralidad. Para la creación de medios fuertemente quirales se requiere que $\kappa^{2}>\mu \varepsilon$.

El concepto de campos paralelos es importante en la formulación teórica del operador y matrices de Dirac [8-9]. De los campos de torsión y la electrodinámica quiral [9-10] y en la Teoría del grafeno como veremos a continuación.

Asumiendo, en las ecuaciones (5)-(7), que $\varepsilon>0$, $\mu>0, \kappa>0 \kappa>0$ y $\kappa>\sqrt{\mu \varepsilon}$, se obtiene que los parámetros $\varepsilon_{D B F}, \mu_{D B F}$ y $T$ son negativos, lo que permite concluir que los medios fuertemente quirales son equivalentes a medios Veselago [11], para ondas polarizadas circularmente a la derecha.

Un caso excepcional se obtiene cuando $\mu=0, \varepsilon=0$ y $\kappa \neq 0$, lo que se traduce, respectivamente, en que $\mu_{D B F}=-\infty, \varepsilon_{D B F}=-\infty$ y $T=-1 / \omega \kappa$. Esta singularidad es conocida como "Chiral Nihility Medium" ([12]), y se caracteriza por tener la interesante propiedad de doble refracción. Si una onda plana polarizada linealmente proveniente del espacio libre e incide sobre un medio de estas características, la onda será dividida en dos ondas polarizadas circularmente, tal que, una de ellas tendrá refracción positiva y la otra refracción negativa, como las obtenidas en los medios Veselago.
En base a las ecuaciones (5)-(7), hemos computado T у $\varepsilon_{\text {II }} / \varepsilon$, o $\mu_{\text {II }} / \mu$ versus $\kappa / \sqrt{\mu \varepsilon}$. Como se muestra en la Figura 2 cuando $\kappa$ es muy cercano a $\sqrt{\mu \varepsilon}$, el valor de $\mathrm{T}$ es muy grande, indicando una fuerte dispersión espacial.

Cuando $0,95<\omega T / c<1,05$, se tiene que $k \equiv \omega / v=w / c(1-w T / c)$ de modo que $v \rightarrow v_{F}$ donde $v_{F}=c(1-\omega T / c)$. Este es el resultado más importante de este trabajo que se desprende del modelo quiral de Dirac para modelar la dinámica de los electrones y huecos en el grafeno.

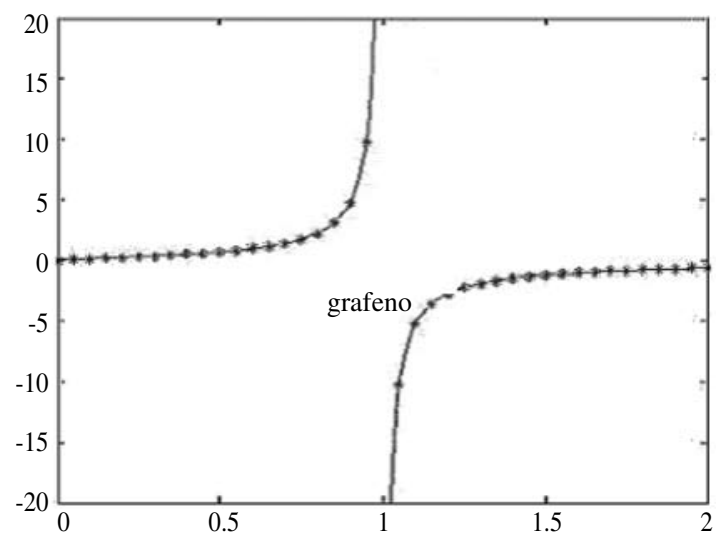

Figura 2. Relación de quiralidad y dispersión espacial. $\omega T / c$ versus $\kappa / \sqrt{\mu \varepsilon}$.

El punto $\kappa / \sqrt{\mu \varepsilon}=1$ es singular, correspondiendo a un valor infinito de T. Cuando $\kappa / \sqrt{\mu \varepsilon}>1$, T se torna negativo. El sistema bidimensional de grafeno puede ser modelado cuando $0,95<\omega T / c<1,05$ y $\kappa / \sqrt{\mu \varepsilon} \sim 1$.

La teoría del grafeno descansa en la hipótesis de que la ecuación de Dirac con masa igual a cero describe correctamente la dinámica del electrón en las bandas de energía con la velocidad de Fermi $v_{F}$ en lugar de la velocidad de la luz c.

$$
H_{0}=-i \hbar v_{F} \sigma \cdot \nabla \Rightarrow-i \hbar v_{F} \sigma \psi=E \psi
$$

Donde $v_{F} \approx 10^{6} \mathrm{~ms}^{-1}$ y $\sigma=\left(\sigma_{x}, \sigma_{y}\right)$ son las matrices de Pauli y $\psi$ es la función de onda en el grafeno. Despreciando efectos de muchos cuerpos esta descripción es teórica y experimentalmente bien descrita [1-2] que indica que el espectro de energía es lineal. El hecho de que los portadores de carga en el grafeno son bien descritos por la ecuación quiral de Dirac (8) con $c \rightarrow v_{F}$, más bien que 
con la usual ecuación de Schrödinger, puede ser visto como una consecuencia de la estructura de cristal del grafeno, que consiste de dos subredes entrelazadas de carbón.

En la Figura 3 la imagen de la izquierda muestra la estructura de red del grafeno hecha de dos redes triangulares que se interpenetran, $\left(\mathbf{a}_{1}\right.$ y $\mathbf{a}_{2}$ son los vectores unitarios de red y $\delta_{i}, i=1,2,3$ son los vectores más cercanos. A la derecha se tiene la zona de Brillouin correspondiente. Los puntos $K, K^{\prime}$ corresponden al caso de $v=v_{F}$ donde los conos de Dirac están localizados) [15].

En la Figura 4 los vectores $\vec{k}_{i}, \vec{k}_{f}$ se encuentran en el plano bidimensional del grafeno, el vector $k_{\gamma}$ del fotón quiral es libre de moverse en cualquier dirección.

Los puntos negros de la Figura 4 a la izquierda, indican la posición de los átomos de carbono.

Se puede usar una solución de dos componentes para demostrar que la quiralidad intrínseca del campo electromagnético explica correctamente que la velocidad de la luz c, en la genuina ecuación de Dirac, es reemplazada por la velocidad de Fermi $c \rightarrow v_{F}$ en un sustrato de grafeno, o sea, los electrones son lentos pero la ecuación en sí es de carácter relativista con quiralidad T. En nuestra derivación asumimos el principio de_Heisenberg como $p T= \pm \hbar / 2$, donde $p_{i, f}=\hbar k_{i, f}$ (Figura 3 ), es el momento linear de las cuasi partículas en la película de grafeno. En la ecuación (8) las funciones de onda $\psi \Rightarrow \psi_{L}=\psi_{\vec{E}}=\vec{\sigma} \cdot \mathbf{E}$ y $\psi \Rightarrow \psi_{R}=\psi_{\vec{H}}=\vec{\sigma} \cdot \boldsymbol{H}^{\bullet}$ donde $\psi_{L, R}$ son las funciones de ondas polarizadas a la izquierda y a la derecha respectivamente, $\mathbf{E}, \boldsymbol{H}^{\bullet}$ corresponden al campo eléctrico y magnético conjugado respectivamente. Se tiene entonces que la ecuación puede ser expresada como:

$$
-i \hbar v_{F} \cdot \sigma\left(\begin{array}{c}
\psi_{L} \\
\psi_{R}
\end{array}\right) \psi=E\left(\begin{array}{l}
\psi_{L} \\
\psi_{R}
\end{array}\right)
$$

Esta ecuación es válida en la red tipo colmena del grafeno, que permite el estudio de efectos cuánticos quirales, la paradoja de Klein, el fenómeno de resistencia negativa en una placa de grafeno cuando se dispone de un voltaje externo, etc. [13-16].

$\mathrm{Al}$ analizar el interesante caso de resistencia negativa, con un voltaje externo aplicado $V$, en un borde del sistema bidimensional, (Figura 5), la expresión (9) se transforma en:

$$
\begin{aligned}
& -i \hbar v_{F} \cdot \sigma\left(\begin{array}{l}
\psi_{L} \\
\psi_{R}
\end{array}\right) \psi=(E-e V)\left(\begin{array}{l}
\psi_{L} \\
\psi_{R}
\end{array}\right), x<0 \\
& -i \hbar v_{F} \cdot \sigma\left(\begin{array}{l}
\psi_{L} \\
\psi_{R}
\end{array}\right) \psi=\left(V_{0}+e V-E\right)\left(\begin{array}{l}
\psi_{L} \\
\psi_{R}
\end{array}\right), D>0
\end{aligned}
$$

En la Figura 6 se observan las características V-I de una barrera energética en el grafeno para $\mathrm{D}=100 \mathrm{~nm}$. En el rango de voltaje de 0,2 a 0,4 se tiene una pronunciada resistencia diferencial negativa, importante en el diseño de osciladores de muy alta frecuencia, con frecuencia de corte $f_{c}=v_{F} / 2 \pi D \sim 1.5 T H z$ para $\mathrm{D}=100 \mathrm{~nm} .100 \mathrm{~nm}$. Como la velocidad de Fermi es mucho menor que la velocidad de la luz, la curva en escalera de la conductividad eléctrica no se distingue.
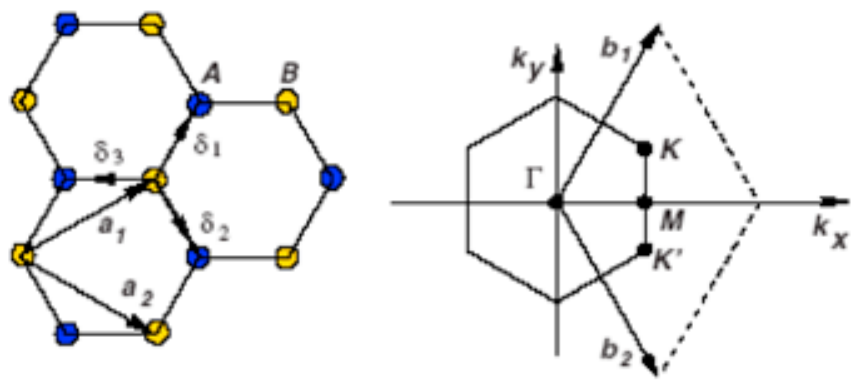

Figura 3. Red tipo colmena y su zona de Brillouin. Fuente: [15]. 


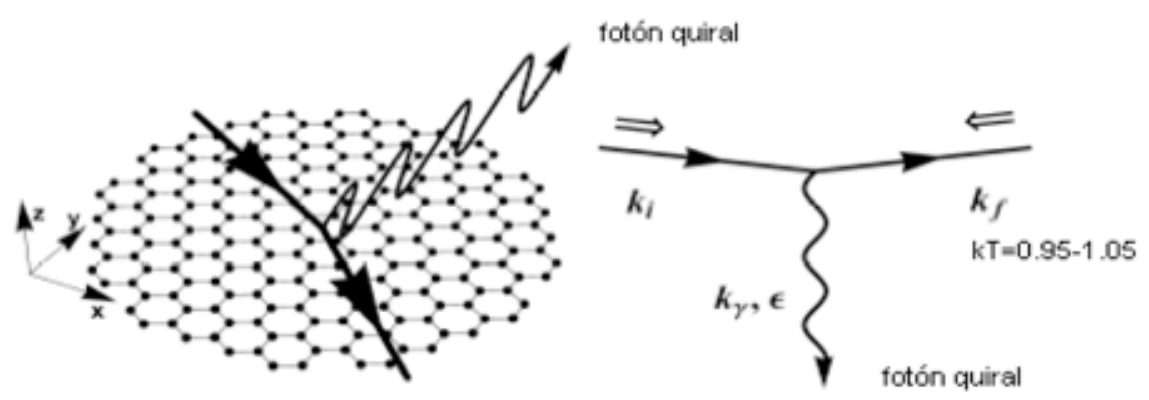

Figura 4. Diagrama donde el grafeno está localizado en el plano (x,y), el fotón quiral tiene cualquier dirección.

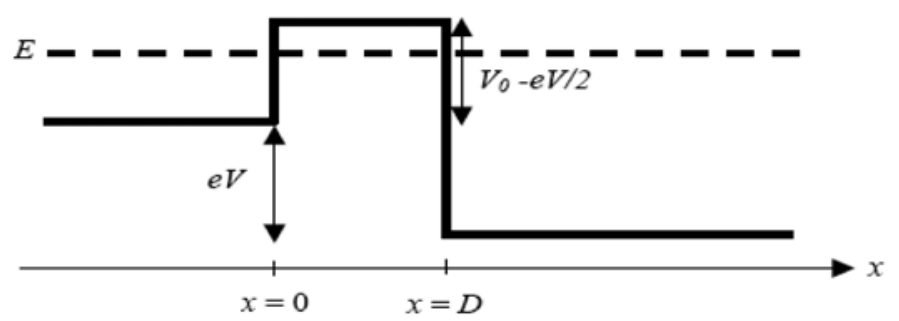

Figura 5. Diagrama de banda de energía para una placa de grafeno polarizado.

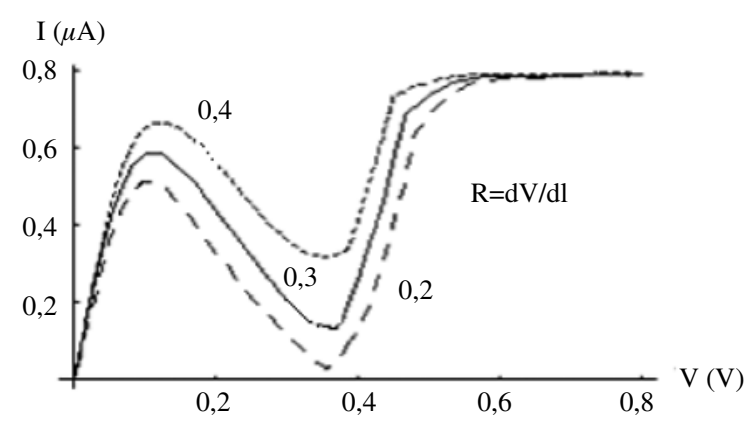

Figura 6. Las curvas V-I voltaje corriente de una barrera en el grafeno para diferentes números de onda de Fermi: $k_{F}=0,2 k_{F 0}$, (línea punteada) $k_{F}=0,3 k_{F 0}$ (línea sólida) $k_{F}=0,4 k_{F 0}$ (línea con trazos).

En el rango de voltaje de 0,2 a 0,4 se tienen valores de resistencia negativa.

\section{CONCLUSIONES}

En este trabajo, son estudiados aspectos teóricos de la quiralidad electromagnética en el grafeno, diferentes a los discutidos en las referencias [1-6] que son de naturaleza geométrica. Específicamente se desarrolló una teoría quiral para el grafeno vinculando las relaciones constitutivas de Born Fedorov de un campo electromagnético con las funciones de onda de la ecuación quiral de Dirac para partículas sin masa. Sus futuras aplicaciones en la nanoelectrónica son amplias y variadas. Como una aplicación específica se dan algunos resultados sobre resistencia diferencial negativa, importante en el diseño de osciladores de muy alta frecuencia.

\section{REFERENCIAS}

[1] K.S. Novoselov, A.K. Geim, S.V. Morozov, D. Jiang, M.I. Katsnelson, I.V. Grigorieva, S.V. Dubonos and A.A. Firsov. "Two-dimensional 
gas of massless Dirac fermions in Graphene". Nature. Vol. 438, pp. 197-200. 2005.

[2] J.T. Sánchez. "Grafeno: sueño o realidad de la nanotecnología". Anales de Mecánica y Electricidad, pp. 40-44. 2010.

[3] N.M.R. Peres. "The Transport properties of graphene: An introduction". Reviews of Modern Physics. Vol. 82, pp. 2673-2700. 2010. DOI: 10.1103/RevModPhys.82.2673

[4] S. Iijima. "Helical microtubules of graphitic carbon". Nature. Vol. 354, p. 56. 1991.

[5] M.M. Fogler, A.H. Castro Neto and F. Guinea. "Effect of external conditions on the structure of scrolled graphene edges". Physical Review B. 81, 161408(R). 2010. URL: http://prb. aps.org/pdf/ PRB/v81/i16/e161408. DOI: 10.1103/PhysRevB. 81.161408.

[6] Lei Liao, Yung-Chen Lin, Mingqiang Bao, Rui Cheng, Jingwei Bai, Yuan Liu, Yongquan $\mathrm{Qu}$, Kang L. Wang, Yu Huang and Xiangfeng Duan. "High-speed graphene transistors with a self-aligned nanowire gate". Nature. Vol. 467, pp. 305-308. 2010.

[7] H. Torres-Silva. "The New Unification of Gravitation and Electromagnetism". ISBN: 978-956-332-973-5. Press Andros. 2011.

[8] H. Torres-Silva. "The Close Relation between the Maxwell System and the Dirac Equation when the Electric Field is Parallel to the Magnetic Field". Ingeniare. Revista chilena de ingeniería. Vol. 16 número especial, pp. 43-47. 2008. DOI: 10.4067/S071833052008000400006

[9] H. Torres-Silva. "Dirac Matrices in Chiral Representation and the Connection with the Electric Field Parallel to the Magnetic Field". Ingeniare. Revista chilena de ingeniería.
Vol. 16 número especial, pp. 48-52. 2008. DOI: 10.4067/S0718-33052008000400007

[10] H. Torres-Silva, A. Vivas-Hernández and I.J. Guerrero-Moreno. "Chiral waves in metamaterial medium". International Journal of pure and Applied Sciences and Technology". Vol. 2 Issue 2, pp. 54-65. ISSN: 2229-6109. 2011.

[11] S. Tretyakov, I. Nefedov, A. Sihvola, S. Maslovski and C. Simovski. "Waves and Energy in Chiral Nihility". J. Electromagnics Waves Appl. Vol. 17 No 5, pp. 695-706. 2003.

[12] C. Zhang and T.J. Cui. Spatial Dispersion and Energy in a Strong Chiral Medium, Optics Express. Vol. 15 No 8, pp. 5114-5119. 2007.

[13] D. Dragoman and M. Dragoman. "Negative differencial resistance of electrons in graphene barrier". Applied Physics Letters. Vol. 90 Issue 14, p. 143111. ISSN: 0003-6951. April 2007. DOI: $10.1063 / 1.2719670$.

[14] M. Koshino and T. Ando. "Transport in bilayer graphene: Calculations within a self-consistent Born approximation”. Phys. Rev. B. 73. 245403. 2006. URL: http://link. aps.org/doi/ 10.1103/PhysRevB.73.245403. DOI: 10.1103/ PhysRevB.73.245403.

[15] A.H. Castro Neto, F. Guinea, N.M. Peres, K.S. Novoselov and A.K. Geim. "The electronic properties of graphene". Rev. Mod. Phys. Vol. 81, p. 109-162. 2009.

[16] R. Nair, P. Blake, A.N. Grigorenko, K.S. Novoselov, T.J. Booth, T. Stauber, N.M.R. Peres and A. K. Geim. "Fine Structure Constant Defines Visual Transparency of Graphene”. Science. Vol. 320, p. 1308. 2008. 